\title{
Improving the Length of Confidence Sets for the Date of a Break in Level and Trend when the Order of Integration is Unknown*
}

\author{
David I. Harvey and Stephen J. Leybourne \\ School of Economics, University of Nottingham
}

May 2016

\begin{abstract}
Harvey and Leybourne (2015) construct confidence sets for the timing of a break in level and/or trend, based on inverting sequences of test statistics for a break at all possible dates. These are valid, in the sense of yielding correct asymptotic coverage, for $I(0)$ or $I(1)$ errors. In constructing the tests, location-dependent weights are chosen for values of the break magnitude parameter such that each test conveniently has the same limit null distribution. By not imposing such a scheme, we show that it is generally possible to significantly shorten the length of the confidence sets, whilst maintaining accurate coverage properties.
\end{abstract}

Keywords: Level break; Trend break; Stationary; Unit root; Confidence sets.

JEL Classification: C22.

\section{Introduction}

Harvey and Leybourne (2015) [HL] propose methods for constructing confidence sets for the date of a break in level and/or trend that are robust to both $I(0)$ and $I(1)$ errors. These are based on the approach of Elliott and Müller (2007) [EM] which involves inverting a sequence of tests for a break at all possible dates. HL derive locally best invariant [LBI] tests separately for when the model errors are $I(0)$ and $I(1)$, with resulting confidence sets providing correct asymptotic coverage regardless of the magnitude of the break. They then suggest using a unit root pre-test procedure to select between the $I(0)$ - and $I(1)$-based confidence sets.

The individual test statistics considered by HL are constructed to maximize an average power criterion, subject to a chosen probability measure for the break magnitude at each date. Following EM, HL use a probability measure that, while not indefensible, is essentially chosen for its expediency, in that under the null of a correct break date, each of the statistics has the same limit null distribution, so that the same asymptotic critical value applies for every assumed break location. Kurozumi and Yamamoto (2015) [KY], in the context of an $I(0)$ model similar to that considered by EM where a break occurs in the coefficients on stationary regressors, argue that this is unnatural in that such a weighting scheme is not motivated by power considerations. Moreover, the EM probability measure for the break magnitude implicitly attributes different weights to breaks occurring at different timings. $\mathrm{KY}$ adopt a more natural probability measure that does not enforce such an artificial structure on the testing problem, and find that this can deliver power gains relative to the EM approach, which translates into a reduction in confidence set length. The HL method for constructing confidence sets for the date of a break in level and/or trend in the presence of $I(1)$ errors relies on first differencing, and bears a close resemblance to the EM and KY model framework. It would be expected, therefore,

${ }^{*}$ Correspondence to: David Harvey, School of Economics, University of Nottingham, Nottingham, NG7 2RD, UK. E-mail: dave.harvey@nottingham.ac.uk 
that use of a KY-type probability measure might result in increased test power and shorter confidence sets, at least in the $I(1)$ context. In this paper, we pursue such a modification of HL. The new weighting scheme results in new limit distributions for both the $I(0)$ - and $I(1)$-based tests, and hence new critical values, which are now location dependent. Using finite sample Monte Carlo simulations, we then show that this new weighting scheme, while having little effect on coverage rates, can yield a significant shortening of the confidence intervals, particularly when the errors are $I(1)$, or are $I(0)$ but exhibit a reasonable degree of persistence.

\section{The model and confidence sets}

As in HL we consider a model for $y_{t}$ that permits a level and/or a trend break in the presence of $I(0)$ or $I(1)$ errors:

$$
\begin{aligned}
& y_{t}=\beta_{1}+\beta_{2} t+\delta_{1} 1\left(t>\left\lfloor\tau_{0} T\right\rfloor\right)+\delta_{2}\left(t-\left\lfloor\tau_{0} T\right\rfloor\right) 1\left(t>\left\lfloor\tau_{0} T\right\rfloor\right)+\varepsilon_{t}, \quad t=1, \ldots, T \\
& \varepsilon_{t}=\rho \varepsilon_{t-1}+u_{t}, \quad t=2, \ldots, T, \quad \varepsilon_{1}=u_{1}
\end{aligned}
$$

with $\left\lfloor\tau_{0} T\right\rfloor \in\{2, \ldots, T-2\} \equiv \Lambda_{T}$ the level and/or trend break point with (unknown) associated break fraction $\tau_{0}$ (' $\lfloor\cdot\rfloor$ ' denoting integer part). In (1), a level break occurs at time $\left\lfloor\tau_{0} T\right\rfloor$ when $\delta_{1} \neq 0$; likewise, a trend break occurs if $\delta_{2} \neq 0$. In $(2)|\rho| \leq 1$ and $u_{t}$ is $I(0)$.

For an assumed break point $\lfloor\tau T\rfloor \in \Lambda_{T}$, we test the null hypothesis $H_{0}:\left\lfloor\tau_{0} T\right\rfloor=\lfloor\tau T\rfloor$ against the alternative $H_{1}:\left\lfloor\tau_{0} T\right\rfloor \neq\lfloor\tau T\rfloor$. Then, following EM, a $(1-\alpha)$-level confidence set for $\tau_{0}$ is constructed by inverting a sequence of $\alpha$-level tests of $H_{0}$ for $\lfloor\tau T\rfloor \in \Lambda_{T}$, with the resulting confidence set comprised of all $\lfloor\tau T\rfloor$ for which $H_{0}$ is not rejected. Provided the test of $H_{0}$ has size $\alpha$ for all $\lfloor\tau T\rfloor$, the confidence set will have correct coverage, as the probability of excluding $\tau_{0}$ from the confidence set is $\alpha$. The more powerful a test is under $H_{1}$ (other things equal), the shorter the resulting confidence set should be.

\section{$3 \quad$ LBI tests}

Under an assumption of $u_{t} \sim \operatorname{NIID}\left(0, \sigma_{u}^{2}\right)$, HL derive LBI tests of $H_{0}$ for the cases where $\rho=0$ and $\rho=1$. These tests are invariant to the unknown parameters $\beta_{1}, \beta_{2}, \delta_{1}$ and $\delta_{2}$ under the null, and can be written as follows, for $I(d)$ errors, $d=0,1$ :

$$
S_{d}(\tau)=\sum_{\lfloor\eta T\rfloor \in \Lambda_{T},\lfloor\eta T\rfloor \neq\lfloor\tau T\rfloor} \hat{u}_{d}^{\prime} D_{d, \eta} H_{d,\lfloor\eta T\rfloor} D_{d, \eta}^{\prime} \hat{u}_{d}
$$

where $D_{0, \eta}$ and $D_{1, \eta}$ are matrices with $t$ th row $d_{0, \eta, t}=[1(t>\lfloor\eta T\rfloor)(t-\lfloor\eta T\rfloor) 1(t>\lfloor\eta T\rfloor)]$ and $d_{1, \eta, t}=[1(t=\lfloor\eta T\rfloor+1) 1(t>\lfloor\eta T\rfloor)]$ respectively, and where $\hat{u}_{0}$ and $\hat{u}_{1}$ denote the OLS residuals from the regressions

$$
y_{t}=\beta_{1}+\beta_{2} t+\delta_{1} 1(t>\lfloor\tau T\rfloor)+\delta_{2}(t-\lfloor\tau T\rfloor) 1(t>\lfloor\tau T\rfloor)+u_{0, t}, \quad t=1, \ldots, T
$$

and

$$
\Delta y_{t}=\beta_{2}+\delta_{1} 1(t=\lfloor\tau T\rfloor+1)+\delta_{2} 1(t>\lfloor\tau T\rfloor)+u_{1, t}, \quad t=2, \ldots, T
$$

respectively. The LBI tests maximize an average power criterion, using a probability measure of $N\left(0, b^{2} H_{d,\lfloor\eta T\rfloor}\right)$ for the break magnitude, with the tests maximizing average power with respect to $b^{2}$ in the locality of $b^{2}=0$ for a given $H_{d,\lfloor\eta T\rfloor}$; see HL for more details. 


\subsection{Selection of $H_{d,\lfloor\eta T\rfloor}$}

As is clear from (3), the form of the LBI test will depend on the specific choice of $H_{d,\lfloor\eta T\rfloor}$. HL specify $H_{d,\lfloor\eta T\rfloor}$ separately for $d=0$ and $d=1$, using

$$
H_{d,\lfloor\eta T\rfloor}= \begin{cases}\operatorname{diag}\left(\lfloor\tau T\rfloor^{-(2-d)},\lfloor\tau T\rfloor^{-2(2-d)}\right) & \text { if }\lfloor\eta T\rfloor<\lfloor\tau T\rfloor \\ \operatorname{diag}\left((T-\lfloor\tau T\rfloor)^{-(2-d)},(T-\lfloor\tau T\rfloor)^{-2(2-d)}\right) & \text { if }\lfloor\eta T\rfloor>\lfloor\tau T\rfloor .\end{cases}
$$

This yields the two statistics

$$
S_{d}(\tau)=\lfloor\tau T\rfloor^{-(2-d)} p_{d, 1, T}+\lfloor\tau T\rfloor^{-2(2-d)} p_{d, 2, T}+(T-\lfloor\tau T\rfloor)^{-(2-d)} p_{d, 1, T}^{\prime}+(T-\lfloor\tau T\rfloor)^{-2(2-d)} p_{d, 2, T}^{\prime}
$$

where

$$
\begin{array}{ll}
p_{0,1, T}=\sum_{t=2}^{\lfloor\tau T-1}\left(\sum_{s=1}^{t} \hat{u}_{0, s}\right)^{2} & p_{0,2, T}=\sum_{t=2}^{\lfloor\tau T\rfloor-1}\left(\sum_{s=1}^{t}(s-t) \hat{u}_{0, s}\right)^{2} \\
p_{0,1, T}^{\prime}=\sum_{t=\lfloor\tau T\rfloor+1}^{T-2}\left(\sum_{s=\lfloor\tau T\rfloor+1}^{t} \hat{u}_{0, s}\right)^{2} & p_{0,2, T}^{\prime}=\sum_{t=\lfloor\tau T\rfloor+1}^{T-2}\left(\sum_{s=\lfloor\tau T\rfloor+1}^{t}(s-t) \hat{u}_{0, s}\right)^{2}
\end{array}
$$

and

$$
\begin{array}{ll}
p_{1,1, T}=\sum_{t=2}^{\lfloor\tau T-1} \hat{u}_{1, t+1}^{2} & p_{1,2, T}=\sum_{t=2}^{\lfloor\tau T\rfloor-1}\left(\sum_{s=2}^{t} \hat{u}_{1, s}\right)^{2} \\
p_{1,1, T}^{\prime}=\sum_{t=\lfloor\tau T\rfloor+1}^{T-2} \hat{u}_{1, t+1}^{2} & p_{1,2, T}^{\prime}=\sum_{t=\lfloor\tau T\rfloor+1}^{T-2}\left(\sum_{s=\lfloor\tau T\rfloor+1}^{t} \hat{u}_{1, s}\right)^{2} .
\end{array}
$$

The standardisations in terms of $d$-dependent powers of $T$ embodied in (4) is unequivocal, as they are the scalings necessary for $S_{0}(\tau)$ and $S_{1}(\tau)$ to be well-behaved in the limit when $|\rho|<1$ and $\rho=1$, respectively, under $H_{0}$. The use of $\tau$-dependent break magnitude probability measure weights, which (essentially) corresponds to scaling $T$ by $\tau$ or $(1-\tau)$, is simply a convenience measure adopted by HL, adapting from EM, to obtain null limiting distributions that do not depend on $\tau$, making tabulation of asymptotic null critical values straightforward. However, there is no other compelling reason to adopt the $\tau$-dependent specification of (4). In particular, (4) is not chosen with any regard to the subsequent power properties of the tests under $H_{1}$. Furthermore, the dependence of the break magnitude weights on the break location seems hardly justified, and in a related context, KY demonstrate that such dependence can reduce test power. We therefore consider an alternative simpler specification for $H_{d,\lfloor\eta T\rfloor}$, along the lines of KY, where break location dependence is not featured:

$$
H_{d,\lfloor\eta T\rfloor}=\operatorname{diag}\left(T^{-(2-d)}, T^{-2(2-d)}\right) \quad \forall\lfloor\eta T\rfloor .
$$

This specification gives rise to two new statistics

$$
S_{d}^{*}(\tau)=T^{-(2-d)} p_{d, 1, T}+T^{-2(2-d)} p_{d, 2, T}+T^{-(2-d)} p_{d, 1, T}^{\prime}+T^{-2(2-d)} p_{d, 2, T}^{\prime} .
$$

\section{Asymptotic distribution of tests}

The statistics considered in the previous section are the LBI tests for $\rho=0$ and $\rho=1$. It is important to stress, however, that $S_{0}^{*}(\tau)$ will be also be a suitable statistic for any $|\rho|<1$, cf. the classic Grenander and Rosenblatt (1957) result demonstrating the asymptotic equivalence of OLS and GLS estimators of coefficients on deterministic terms in an $I(0)$ series. Moreover, as we show below, $S_{0}^{*}(\tau)$ has the same null limit distribution for any $|\rho|<1$.

For our asymptotic results, we adopt the two assumptions from HL, which pertain to the $I(0)$ case of $|\rho|<1$, and the $I(1)$ case of $\rho=1$, and permit serial correlation in $u_{t}$. Under $H_{0}$, we have:

(a) $I(0)$ : Let $|\rho|<1, u_{t}=C(L) \zeta_{t}, C(L)=\sum_{i=0}^{\infty} C_{i} L^{i}, C_{0}=1$, with $C(z) \neq 0$ for all $|z| \leq 1$ and $\sum_{i=0}^{\infty} i\left|C_{i}\right|<\infty$, and where $\zeta_{t}$ is an $I I D$ sequence with mean zero, variance $\sigma^{2}$ and finite fourth moment. Let $\omega_{u}^{2}=\lim _{T \rightarrow \infty} T^{-1} E\left(\sum_{t=1}^{T} u_{t}\right)^{2}=\sigma^{2} C(1)^{2}$ and $\omega_{\varepsilon}^{2}=\omega_{u}^{2} /(1-\rho)^{2}$. Then

$\omega_{\varepsilon}^{-2} S_{0}^{*}(\tau) \stackrel{d}{\rightarrow} \tau^{2} \int_{0}^{1} B_{2}(r)^{2} d r+\tau^{4} \int_{0}^{1} K(r)^{2} d r+(1-\tau)^{2} \int_{0}^{1} B_{2}^{\prime}(r)^{2} d r+(1-\tau)^{4} \int_{0}^{1} K^{\prime}(r)^{2} d r \equiv \mathcal{L}_{0}^{*}(\tau)$. 
(b) $I(1)$ : Let $\rho=1$ with $u_{t}$ defined as in (a) and $\sigma_{u}^{2}=E\left(u_{t}^{2}\right)$. Then

$$
\omega_{u}^{-2}\left\{S_{1}^{*}(\tau)-\sigma_{u}^{2}\right\} \stackrel{d}{\rightarrow} \tau^{2} \int_{0}^{1} B_{1}(r)^{2} d r+(1-\tau)^{2} \int_{0}^{1} B_{1}^{\prime}(r)^{2} d r \equiv \mathcal{L}_{1}^{*}(\tau) .
$$

Here $B_{1}(r)=B(r)-r B(1), B_{2}(r)=B_{1}(r)+6 r(1-r)\left\{\frac{1}{2} B(1)-\int_{0}^{1} B(s) d s\right\}$ and $K(r)=-r^{2}(1-$ $r) B(1)-\int_{0}^{r} B(s) d s+r^{2}(3-2 r) \int_{0}^{1} B(s) d s$, with $B(r)$ a standard Brownian motion process; $B_{1}^{\prime}(r)$, $B_{2}^{\prime}(r)$ and $K^{\prime}(r)$ take the same forms as $B_{1}(r), B_{2}(r)$ and $K(r)$, respectively, but with $B(r)$ replaced by $B^{\prime}(r)$, with $B^{\prime}(r)$ a Brownian motion independent of $B(r)$. Proofs of these limits are straightforward modifications of those in HL. Notice the centering to $S_{1}^{*}(\tau)$ is $\sigma_{u}^{2}$, as opposed to $2 \sigma_{u}^{2}$ for $S_{1}(\tau)$ in HL; this arises since $T^{-1} p_{1,1, T}+T^{-1} p_{1,1, T}^{\prime}=T^{-1} \sum_{t=2}^{T-2} \hat{u}_{1, t+1}^{2}-T^{-1} \hat{u}_{1,\lfloor\tau T\rfloor+1}^{2} \stackrel{p}{\rightarrow} \sigma_{u}^{2}$.

\subsection{Response surface critical values}

Clearly, $\mathcal{L}_{0}^{*}(\tau)$ and $\mathcal{L}_{1}^{*}(\tau)$ depend on $\tau$. Hence, as in $\mathrm{KY}$, we use a response surface to provide asymptotic null critical values. To accomplish this we simulated (upper tail) $\alpha$-level critical values for the limit distributions $\mathcal{L}_{0}^{*}(\tau)$ and $\mathcal{L}_{1}^{*}(\tau)$. These were obtained by direct simulation of the limiting distributions above for the grid of values $\tau \in\{0.01,0.02, \ldots, 0.99\}$, approximating the Brownian motion processes using $\operatorname{NIID}(0,1)$ random variates, and with the integrals approximated by normalized sums of 2000 steps, and using 50,000 Monte Carlo replications. Denoting a simulated critical value as $c v(\tau)$ we then ran the discretised OLS regression

$$
c v(\tau)=b_{0}+b_{1}\{h(\tau)+1\}^{-1}+b_{2} h(\tau)+b_{3} h(\tau)^{2}+b_{4} h(\tau)^{3}+\text { error }
$$

with $h(\tau)=|\tau-0.5|$, adopting the functional form used in KY. The parameter estimates are shown in Table 1 for $\mathcal{L}_{0}^{*}(\tau)$ and $\mathcal{L}_{1}^{*}(\tau)$ and $\alpha=0.01,0.05,0.10$ and for each regression we find $R^{2}>0.9995$. Hence, if the fitted critical values are applied to each of the sequence of tests $\omega_{\varepsilon}^{-2} S_{0}^{*}(\tau)$ under $I(0)$ errors, and $\omega_{u}^{-2}\left\{S_{1}^{*}(\tau)-\sigma_{u}^{2}\right\}$ under $I(1)$ errors across $\tau$, the corresponding confidence set based on inverting these tests will have asymptotically correct coverage.

Table 1. Response surface parameter estimates

\begin{tabular}{rrrrrrrr}
\hline & \multicolumn{3}{c}{$\mathcal{L}_{0}^{*}(\tau)$} & & \multicolumn{3}{c}{$\mathcal{L}_{1}^{*}(\tau)$} \\
\cline { 2 - 4 } \cline { 6 - 8 }$\alpha$ & \multicolumn{1}{c}{0.01} & 0.05 & 0.10 & & 0.01 & \multicolumn{1}{c}{0.05} & \multicolumn{1}{c}{0.10} \\
\hline$b_{0}$ & -2.9691 & -0.9281 & -0.4327 & & -13.1047 & -5.0587 & -1.7865 \\
$b_{1}$ & 3.0526 & 0.9904 & 0.4860 & & 13.3702 & 5.2467 & 1.9390 \\
$b_{2}$ & 3.0554 & 0.9877 & 0.4777 & & 13.3837 & 5.1849 & 1.8939 \\
$b_{3}$ & -1.9424 & -0.3988 & -0.0632 & & -9.3322 & -2.9697 & -0.5644 \\
$b_{4}$ & 0.9882 & 0.2333 & 0.0787 & & 4.5264 & 1.3809 & 0.2931 \\
\hline
\end{tabular}

\section{$5 \quad$ Feasible tests and confidence set selection}

Feasible variants of $S_{0}^{*}(\tau)$ and $S_{1}^{*}(\tau)$ require an estimator of $\omega_{\varepsilon}^{2}$ for the former, and $\omega_{u}^{2}$ and $\sigma_{u}^{2}$ for the latter. We employ the same estimators as favoured by HL in the context of $S_{0}(\tau)$ and $S_{1}(\tau)$. These are the estimators $\hat{\omega}_{\varepsilon, P}^{2}\left(\hat{\tau}_{D_{m}}\right), \hat{\omega}_{u, P}^{2}\left(\hat{\tau}_{D_{m}}\right)$ and $\hat{\sigma}_{u}^{2}\left(\hat{\tau}_{D_{m}}\right)$ of that paper. The first two are Berktype parametric autoregressive spectral density estimators. Each of the three estimators is based on residuals from a regression that incorporates a level/trend break fitted at same estimated break fraction $\hat{\tau}_{D_{m}}$, where $\hat{\tau}_{D_{m}}$ is the estimator of $\tau_{0}$ suggested by Harvey and Leybourne (2014). More detail of the construction of this estimator can be found in HL, section 3.2. The feasible tests are then

$$
\hat{S}_{0, P}^{* \hat{\tau}_{P}}(\tau)=\hat{\omega}_{\varepsilon, P}^{-2}\left(\hat{\tau}_{D_{m}}\right) S_{0}^{*}(\tau), \quad \hat{S}_{1, P}^{* \hat{\tau}_{P}}(\tau)=\hat{\omega}_{u, P}^{-2}\left(\hat{\tau}_{D_{m}}\right)\left\{S_{1}^{*}(\tau)-\hat{\sigma}_{u}^{2}\left(\hat{\tau}_{D_{m}}\right)\right\} .
$$


In practice, the order of integration of the errors is unknown, and therefore a method is required to be able to choose between the $I(0)$-based confidence set associated with $\hat{S}_{0, P}^{* \hat{\tau}}(\tau)$ and the $I(1)$-based confidence set for $\hat{S}_{1, P}^{* \hat{\tau}}(\tau)$. In line with HL, section 4 , we employ a pre-test for the null of $\rho=1$ against the alternative of $|\rho|<1$, which is robust to the possible presence of a break in level and trend. Practically, this involves running the left-tailed unit root single break MDF test of Harvey et al. (2013), then selecting the $\hat{S}_{0, P}^{* \hat{\tau}_{2}}(\tau)$ confidence set if $M D F<c v_{\alpha}$ and the $\hat{S}_{1, P}^{* \hat{\tau}_{P}}(\tau)$ confidence set if $M D F \geq c v_{\alpha}$, where $c v_{\alpha}$ denotes the asymptotic $\alpha$-level unit root null critical value of $M D F$. We denote this pre-test based procedure as $\hat{S}_{\text {pre,P }}^{* \hat{r}}(\tau)$.

\section{$6 \quad$ Finite sample comparisons}

We now examine how confidence sets which are based on $\hat{S}_{p r e, P}^{* \hat{\tau}}(\tau)$ compare with those of $\hat{S}_{p r e, P}^{\hat{\tau}}(\tau)$, its counterpart from HL. In terms of their construction, it is important to remember the only difference between the new tests and their forebears in HL lies in the modification to $H_{d,\lfloor\eta T\rfloor}$ (and the consequent change to the $\hat{\sigma}_{u}^{2}\left(\hat{\tau}_{D_{m}}\right)$ centering in $\left.\hat{S}_{1, P}^{* \hat{\tau}}(\tau)\right)$. As regards other settings relevant to both sets of tests, the number of lagged difference terms in the fitted autoregressions that underpin $\hat{\omega}_{\varepsilon, P}^{2}\left(\hat{\tau}_{D_{m}}\right)$ and $\hat{\omega}_{u, P}^{2}\left(\hat{\tau}_{D_{m}}\right)$ is selected via the BIC with maximum value $\ell_{\max }=\left\lfloor 12(T / 100)^{1 / 4}\right\rfloor$. The same value $\ell_{\max }$ is employed by the MAIC procedure of Perron and $\mathrm{Qu}(2007)$ to determine the length used by the unit root test $M D F$. We adopt a 0.10 trimming for allowable break locations such that $\lfloor\tau T\rfloor \in$ $\{\lfloor 0.1 T\rfloor, \ldots,\lfloor 0.9 T\rfloor\}$; this same trimming is also imposed when constructing $\hat{\tau}_{D_{m}}$ and $M D F$. Each test (including $M D F$ ) is conducted at the 0.05 -level using the appropriate asymptotic critical value.

We simulate the DGP (1)-(2) with $\beta_{1}=\beta_{2}=0$ (without loss of generality) using $u_{t} \sim N I I D(0,1)$. The values of $\rho$ we consider for $\varepsilon_{t}$ are $\rho \in\{0.00,0.50,0.80,0.90,0.95,1.00\}$ to encompass a range of $I(0)$ processes and an $I(1)$ process. As regards the break timings we use $\tau_{0} \in\{0.3,0.5,0.7\}$, corresponding to early, middle and late sample breaks. The constellations we adopt for break magnitudes are $\left(\delta_{1}, \delta_{2}\right) \in\left\{\left(3 c_{1}, 0.3 c_{2}\right),\left(4 c_{1}, 0.4 c_{2}\right),\left(5 c_{1}, 0.5 c_{2}\right),\left(6 c_{1}, 0.6 c_{2}\right)\right\}$ with $c_{1}=c_{2}=1$ representing a break in both level and trend (Table 2); $c_{1}=1, c_{2}=0$ a break in level alone (Table 3 ); $c_{1}=0, c_{2}=1$ a break in trend alone (Table 4). Sample sizes are set at $T=150$ and $T=300$.

All simulations are performed using 10,000 Monte Carlo replications, and we report results for confidence set coverage (the proportion of replications for which the true break date is contained in the confidence set) and confidence set length (in each replication, length is calculated as the number of dates included in the confidence set as a proportion of the sample size; we then report the average length over Monte Carlo replications). In what follows, we adopt a shorthand notation using $S$ and $S^{*}$ to denote $\hat{S}_{p r e, P}^{\hat{\tau}}(\tau)$ and $\hat{S}_{p r e, P}^{* \hat{\tau}}(\tau)$, respectively.

Consider first the coverage rates of $S$ and $S^{*}$. Tables 2-4 show that there is very little to choose between $S$ and $S^{*}$ in terms of their levels of accuracy, and in general, both tests deliver coverage rates either close to the nominal level or higher. When $\rho=1.00$ and a break of small magnitude is present, both tests suffer from some degree of under-coverage, particularly when only a trend break occurs. Here, $S^{*}$ can exhibit slightly more under-coverage than $S$ when $\tau_{0}=0.50$, although for $T=300$ the differences are small. The reverse pattern is true when $\rho=0.00$ and only a trend break occurs, with $S$ often being slightly under-sized while $S^{*}$ retains coverage close to 0.95 . Overall, the picture is one of decent coverage across the different settings, with little difference between $S$ and $S^{*}$.

We now turn to comparing the confidence set lengths. In Table 2 , when $T=150$ and $\rho=0.00$ or $\rho=0.50, S^{*}$ generally yields the shorter lengths for $\tau_{0}=0.3$ and $\tau_{0}=0.5$, while $S$ yields the shorter lengths for $\tau_{0}=0.7$. The differences are small, however, as both procedures give short confidence sets for these values of $\rho$. For $\rho>0.50$, a systematic ranking emerges, whereby the shorter lengths are always associated with $S^{*}$. Moreover, we observe that the improvements afforded by $S^{*}$ can be substantial. When $\rho>0.80$, it is common that $S^{*}$ produces confidences sets with lengths some 0.15-0.20 shorter than those of $S$. Moving on to $T=300$, as we might predict, there is a general 
overall shortening of all the confidence sets since the individual tests reject more frequently under $H_{1}$. Here, we now see that $S^{*}$ provides systematically shorter confidence sets than $S$ for $\rho>0.80$. The improvements afforded by $S^{*}$ are now commonly in the range of $0.05-0.10$, which is obviously a lower range than for $T=150$, but still not insubstantial.

In Table 3 the lengths for both $S$ and $S^{*}$ are generally larger than in Table 2, as would be expected since the trend break is now absent, lowering rejection frequencies under $H_{1}$. What is also evident is that when $\rho=1.00$, shorter lengths are obtained with $T=150$ than with $T=300$, due to the fact that a fixed magnitude level break is asymptotically undetectable in an $I(1)$ process. We see a similar, but less emphasized, phenomenon with $\rho=0.95$, which might be considered a "near $I(1)$ " process in the current context. Comparing $S$ and $S^{*}$, the two are similar for $\rho=0.00$ or $\rho=0.50$, while $S^{*}$ always yields the shorter confidence set for $\rho>0.50$ for $T=150$ and $T=300$. Shortenings of up to about 0.25 are seen when $T=150$, with many in the range $0.15-0.20$. When $T=300$, the shortenings are less pronounced, but can still comfortably exceed 0.10 in some cases.

The same broad comparison between $S$ and $S^{*}$ also pertains to Table 4, with $S^{*}$ generally providing the shorter lengths for $\rho>0.50$. Interestingly, despite overall lengths here tending to exceed those in Table 2 due to the absence of the level break, the extent to which $S^{*}$ reduces length appears rather less substantial, although gains in the range of 0.05-0.10 do still frequently occur.

In summary then, it is clear that the new procedure $S^{*}$ can result in shorter confidence sets than the original procedure $S$. While there is some ambiguity as to whether any gains from $S^{*}$ are meaningful for small values of $\rho$, for the larger values of $\rho$ they can be considerable (particularly in a model which contains both a level and trend break). From an empirical perspective, that the better gains are made for moderately persistent $I(0)$ processes to highly persistent $I(1)$ processes is of some relevance, as these kinds of persistent series are often encountered in applied macroeconomic and financial time series analysis. At the slight expense of introducing location dependent asymptotic critical values (which are easily made accessible via a response surface), use of $S^{*}$ compared to $S$ can improve length with little impact on coverage rates, and we therefore recommend the modified procedure for empirical work.

\section{References}

Elliott, G. and Müller, U.K. (2007). Confidence sets for the date of a single break in linear time series regressions. Journal of Econometrics 141, 1196-1218.

Grenander, U. and Rosenblatt, M. (1957). Statistical Analysis of Stationary Time Series, John Wiley, New York.

Harvey, D.I. and Leybourne, S.J. (2014). Break date estimation for models with deterministic structural change. Oxford Bulletin of Economics and Statistics 76, 623-642.

Harvey, D.I., Leybourne, S.J. and Taylor, A.M.R. (2013). Testing for unit roots in the possible presence of multiple trend breaks using minimum Dickey-Fuller statistics. Journal of Econometrics, $177,265-284$.

Harvey, D.I. and Leybourne, S.J. (2015). Confidence sets for the date of a break in level and trend when the order of integration is unknown. Journal of Econometrics 184, 262-279.

Kurozumi, E. and Yamamoto, Y. (2015). Confidence sets for the break date based on optimal tests. Econometrics Journal 18, 412-435.

Perron, P. and Qu, Z. (2007). A simple modification to improve the finite sample properties of $\mathrm{Ng}$ and Perron's unit root tests. Economics Letters 94, 12-19. 
Table 2. Finite sample coverage and length of nominal 0.95-level confidence sets.

\begin{tabular}{|c|c|c|c|c|c|c|c|c|c|c|c|c|c|c|c|}
\hline \multirow[b]{2}{*}{$T$} & \multirow[b]{2}{*}{$\tau_{0}$} & \multirow[b]{2}{*}{$\delta_{1}$} & \multirow[b]{2}{*}{$\delta_{2}$} & \multicolumn{2}{|c|}{$\rho=0.00$} & \multicolumn{2}{|c|}{$\rho=0.50$} & \multicolumn{2}{|c|}{$\rho=0.80$} & \multicolumn{2}{|c|}{$\rho=0.90$} & \multicolumn{2}{|c|}{$\rho=0.95$} & \multicolumn{2}{|c|}{$\rho=1.00$} \\
\hline & & & & $S$ & $S^{*}$ & $S$ & $S^{*}$ & $S$ & $S^{*}$ & $S$ & $S^{*}$ & $S$ & $S^{*}$ & $S$ & $S^{*}$ \\
\hline \multicolumn{16}{|c|}{ Panel A. Coverage } \\
\hline \multirow[t]{12}{*}{150} & 0.3 & 3 & 0.3 & 0.944 & 0.955 & 0.963 & 0.963 & 0.979 & 0.980 & 0.983 & 0.988 & 0.967 & 0.978 & 0.929 & 0.938 \\
\hline & & 4 & 0.4 & 0.945 & 0.954 & 0.968 & 0.962 & 0.987 & 0.984 & 0.991 & 0.994 & 0.982 & 0.990 & 0.946 & 0.953 \\
\hline & & 5 & 0.5 & 0.945 & 0.953 & 0.971 & 0.961 & 0.992 & 0.983 & 0.995 & 0.995 & 0.987 & 0.993 & 0.956 & 0.962 \\
\hline & & 6 & 0.6 & 0.946 & 0.952 & 0.971 & 0.960 & 0.993 & 0.983 & 0.996 & 0.995 & 0.989 & 0.994 & 0.958 & 0.963 \\
\hline & 0.5 & 3 & 0.3 & 0.950 & 0.951 & 0.965 & 0.966 & 0.981 & 0.982 & 0.982 & 0.983 & 0.976 & 0.974 & 0.935 & 0.934 \\
\hline & & 4 & 0.4 & 0.948 & 0.950 & 0.968 & 0.970 & 0.991 & 0.992 & 0.992 & 0.993 & 0.989 & 0.990 & 0.954 & 0.961 \\
\hline & & 5 & 0.5 & 0.948 & 0.950 & 0.967 & 0.968 & 0.994 & 0.995 & 0.997 & 0.998 & 0.994 & 0.995 & 0.962 & 0.969 \\
\hline & & 6 & 0.6 & 0.947 & 0.949 & 0.968 & 0.969 & 0.995 & 0.996 & 0.998 & 0.999 & 0.995 & 0.996 & 0.964 & 0.971 \\
\hline & 0.7 & 3 & 0.3 & 0.941 & 0.955 & 0.964 & 0.960 & 0.982 & 0.965 & 0.976 & 0.973 & 0.968 & 0.975 & 0.931 & 0.931 \\
\hline & & 4 & 0.4 & 0.943 & 0.954 & 0.968 & 0.961 & 0.991 & 0.975 & 0.988 & 0.983 & 0.981 & 0.988 & 0.949 & 0.951 \\
\hline & & 5 & 0.5 & 0.946 & 0.953 & 0.969 & 0.960 & 0.993 & 0.979 & 0.994 & 0.990 & 0.987 & 0.992 & 0.958 & 0.959 \\
\hline & & 6 & 0.6 & 0.946 & 0.951 & 0.969 & 0.960 & 0.993 & 0.979 & 0.996 & 0.991 & 0.990 & 0.995 & 0.961 & 0.960 \\
\hline \multirow[t]{12}{*}{300} & 0.3 & 3 & 0.3 & 0.946 & 0.949 & 0.957 & 0.955 & 0.982 & 0.965 & 0.991 & 0.981 & 0.992 & 0.994 & 0.941 & 0.942 \\
\hline & & 4 & 0.4 & 0.947 & 0.949 & 0.959 & 0.954 & 0.984 & 0.965 & 0.995 & 0.981 & 0.996 & 0.995 & 0.951 & 0.954 \\
\hline & & 5 & 0.5 & 0.947 & 0.948 & 0.960 & 0.953 & 0.985 & 0.963 & 0.996 & 0.981 & 0.998 & 0.996 & 0.955 & 0.956 \\
\hline & & 6 & 0.6 & 0.947 & 0.949 & 0.960 & 0.953 & 0.985 & 0.963 & 0.996 & 0.981 & 0.998 & 0.996 & 0.956 & 0.956 \\
\hline & 0.5 & 3 & 0.3 & 0.948 & 0.950 & 0.960 & 0.962 & 0.978 & 0.980 & 0.989 & 0.990 & 0.992 & 0.992 & 0.943 & 0.944 \\
\hline & & 4 & 0.4 & 0.947 & 0.949 & 0.959 & 0.961 & 0.980 & 0.983 & 0.993 & 0.994 & 0.997 & 0.997 & 0.954 & 0.958 \\
\hline & & 5 & 0.5 & 0.947 & 0.949 & 0.958 & 0.960 & 0.980 & 0.983 & 0.994 & 0.995 & 0.998 & 0.998 & 0.959 & 0.964 \\
\hline & & 6 & 0.6 & 0.946 & 0.948 & 0.958 & 0.960 & 0.980 & 0.983 & 0.995 & 0.996 & 0.999 & 0.999 & 0.959 & 0.964 \\
\hline & 0.7 & 3 & 0.3 & 0.943 & 0.949 & 0.958 & 0.956 & 0.980 & 0.963 & 0.992 & 0.976 & 0.990 & 0.986 & 0.939 & 0.942 \\
\hline & & 4 & 0.4 & 0.944 & 0.948 & 0.960 & 0.954 & 0.983 & 0.964 & 0.993 & 0.978 & 0.996 & 0.991 & 0.950 & 0.952 \\
\hline & & 5 & 0.5 & 0.946 & 0.947 & 0.960 & 0.953 & 0.983 & 0.965 & 0.995 & 0.981 & 0.997 & 0.993 & 0.955 & 0.956 \\
\hline & & 6 & 0.6 & 0.946 & 0.946 & 0.959 & 0.952 & 0.983 & 0.964 & 0.996 & 0.981 & 0.998 & 0.994 & 0.956 & 0.956 \\
\hline \multicolumn{16}{|c|}{ Panel B. Length } \\
\hline \multirow[t]{12}{*}{150} & 0.3 & 3 & 0.3 & 0.094 & 0.086 & 0.179 & 0.174 & 0.368 & 0.352 & 0.608 & 0.560 & 0.636 & 0.578 & 0.585 & 0.520 \\
\hline & & 4 & 0.4 & 0.054 & 0.047 & 0.142 & 0.133 & 0.289 & 0.254 & 0.467 & 0.356 & 0.493 & 0.371 & 0.459 & 0.350 \\
\hline & & 5 & 0.5 & 0.031 & 0.029 & 0.113 & 0.102 & 0.219 & 0.189 & 0.300 & 0.197 & 0.320 & 0.191 & 0.315 & 0.182 \\
\hline & & 6 & 0.6 & 0.021 & 0.022 & 0.088 & 0.077 & 0.177 & 0.152 & 0.192 & 0.114 & 0.196 & 0.089 & 0.198 & 0.076 \\
\hline & 0.5 & 3 & 0.3 & 0.086 & 0.070 & 0.167 & 0.149 & 0.348 & 0.301 & 0.591 & 0.500 & 0.637 & 0.528 & 0.591 & 0.484 \\
\hline & & 4 & 0.4 & 0.045 & 0.037 & 0.128 & 0.114 & 0.278 & 0.210 & 0.470 & 0.281 & 0.503 & 0.295 & 0.470 & 0.295 \\
\hline & & 5 & 0.5 & 0.026 & 0.023 & 0.097 & 0.086 & 0.209 & 0.156 & 0.304 & 0.136 & 0.322 & 0.123 & 0.314 & 0.127 \\
\hline & & 6 & 0.6 & 0.018 & 0.018 & 0.073 & 0.063 & 0.161 & 0.129 & 0.175 & 0.078 & 0.171 & 0.051 & 0.172 & 0.046 \\
\hline & 0.7 & 3 & 0.3 & 0.075 & 0.104 & 0.156 & 0.162 & 0.343 & 0.310 & 0.582 & 0.505 & 0.622 & 0.534 & 0.579 & 0.490 \\
\hline & & 4 & 0.4 & 0.039 & 0.068 & 0.116 & 0.134 & 0.266 & 0.220 & 0.448 & 0.297 & 0.476 & 0.316 & 0.445 & 0.305 \\
\hline & & 5 & 0.5 & 0.023 & 0.042 & 0.086 & 0.114 & 0.194 & 0.167 & 0.273 & 0.151 & 0.288 & 0.141 & 0.277 & 0.138 \\
\hline & & 6 & 0.6 & 0.017 & 0.027 & 0.063 & 0.096 & 0.146 & 0.142 & 0.144 & 0.089 & 0.138 & 0.060 & 0.138 & 0.051 \\
\hline 300 & 0.3 & 3 & 0.3 & 0.065 & 0.064 & 0.102 & 0.107 & 0.173 & 0.175 & 0.337 & 0.307 & 0.580 & 0.503 & 0.540 & 0.491 \\
\hline & & 4 & 0.4 & 0.045 & 0.042 & 0.087 & 0.089 & 0.142 & 0.145 & 0.238 & 0.220 & 0.380 & 0.308 & 0.396 & 0.336 \\
\hline & & 5 & 0.5 & 0.030 & 0.027 & 0.075 & 0.075 & 0.123 & 0.127 & 0.177 & 0.176 & 0.228 & 0.211 & 0.259 & 0.205 \\
\hline & & 6 & 0.6 & 0.020 & 0.019 & 0.065 & 0.062 & 0.112 & 0.114 & 0.149 & 0.146 & 0.165 & 0.145 & 0.165 & 0.120 \\
\hline & 0.5 & 3 & 0.3 & 0.060 & 0.055 & 0.098 & 0.093 & 0.167 & 0.154 & 0.318 & 0.265 & 0.559 & 0.439 & 0.536 & 0.451 \\
\hline & & 4 & 0.4 & 0.038 & 0.034 & 0.082 & 0.078 & 0.137 & 0.128 & 0.234 & 0.187 & 0.379 & 0.250 & 0.394 & 0.284 \\
\hline & & 5 & 0.5 & 0.023 & 0.021 & 0.069 & 0.065 & 0.119 & 0.112 & 0.177 & 0.146 & 0.241 & 0.151 & 0.261 & 0.149 \\
\hline & & 6 & 0.6 & 0.015 & 0.014 & 0.057 & 0.053 & 0.107 & 0.102 & 0.146 & 0.122 & 0.167 & 0.092 & 0.163 & 0.066 \\
\hline & 0.7 & 3 & 0.3 & 0.051 & 0.073 & 0.094 & 0.102 & 0.165 & 0.162 & 0.322 & 0.285 & 0.560 & 0.471 & 0.537 & 0.472 \\
\hline & & 4 & 0.4 & 0.029 & 0.057 & 0.075 & 0.088 & 0.134 & 0.136 & 0.234 & 0.204 & 0.382 & 0.289 & 0.392 & 0.311 \\
\hline & & 5 & 0.5 & 0.017 & 0.041 & 0.061 & 0.079 & 0.116 & 0.120 & 0.175 & 0.161 & 0.239 & 0.185 & 0.252 & 0.181 \\
\hline & & 6 & 0.6 & 0.012 & 0.027 & 0.048 & 0.071 & 0.103 & 0.109 & 0.141 & 0.134 & 0.156 & 0.122 & 0.148 & 0.096 \\
\hline
\end{tabular}


Table 3. Finite sample coverage and length of nominal 0.95-level confidence sets.

\begin{tabular}{|c|c|c|c|c|c|c|c|c|c|c|c|c|c|c|c|}
\hline \multirow[b]{2}{*}{$T$} & \multirow[b]{2}{*}{$\tau_{0}$} & \multirow[b]{2}{*}{$\delta_{1}$} & \multirow[b]{2}{*}{$\delta_{2}$} & \multicolumn{2}{|c|}{$\rho=0.00$} & \multicolumn{2}{|c|}{$\rho=0.50$} & \multicolumn{2}{|c|}{$\rho=0.80$} & \multicolumn{2}{|c|}{$\rho=0.90$} & \multicolumn{2}{|c|}{$\rho=0.95$} & \multicolumn{2}{|c|}{$\rho=1.00$} \\
\hline & & & & $S$ & $S^{*}$ & $S$ & $S^{*}$ & $S$ & $S^{*}$ & $S$ & $S^{*}$ & $S$ & $S^{*}$ & $S$ & $S^{*}$ \\
\hline \multicolumn{16}{|c|}{ Panel A. Coverage } \\
\hline \multirow[t]{12}{*}{150} & 0.3 & 3 & 0 & 0.939 & 0.959 & 0.960 & 0.958 & 0.947 & 0.928 & 0.951 & 0.936 & 0.954 & 0.960 & 0.912 & 0.919 \\
\hline & & 4 & 0 & 0.943 & 0.959 & 0.969 & 0.964 & 0.977 & 0.961 & 0.976 & 0.967 & 0.971 & 0.976 & 0.936 & 0.943 \\
\hline & & 5 & 0 & 0.944 & 0.960 & 0.971 & 0.965 & 0.991 & 0.975 & 0.990 & 0.985 & 0.983 & 0.987 & 0.951 & 0.956 \\
\hline & & 6 & 0 & 0.944 & 0.961 & 0.972 & 0.965 & 0.994 & 0.980 & 0.996 & 0.992 & 0.987 & 0.992 & 0.956 & 0.960 \\
\hline & 0.5 & 3 & 0 & 0.954 & 0.955 & 0.967 & 0.968 & 0.958 & 0.960 & 0.965 & 0.964 & 0.971 & 0.967 & 0.921 & 0.915 \\
\hline & & 4 & 0 & 0.955 & 0.957 & 0.971 & 0.973 & 0.985 & 0.986 & 0.983 & 0.984 & 0.982 & 0.983 & 0.946 & 0.950 \\
\hline & & 5 & 0 & 0.957 & 0.959 & 0.971 & 0.972 & 0.994 & 0.995 & 0.994 & 0.995 & 0.991 & 0.992 & 0.957 & 0.964 \\
\hline & & 6 & 0 & 0.957 & 0.959 & 0.971 & 0.973 & 0.995 & 0.996 & 0.998 & 0.998 & 0.995 & 0.996 & 0.963 & 0.970 \\
\hline & 0.7 & 3 & 0 & 0.939 & 0.961 & 0.961 & 0.959 & 0.948 & 0.929 & 0.951 & 0.944 & 0.959 & 0.965 & 0.920 & 0.918 \\
\hline & & 4 & 0 & 0.941 & 0.960 & 0.969 & 0.965 & 0.975 & 0.959 & 0.975 & 0.968 & 0.972 & 0.976 & 0.938 & 0.939 \\
\hline & & 5 & 0 & 0.944 & 0.959 & 0.970 & 0.964 & 0.991 & 0.976 & 0.990 & 0.985 & 0.984 & 0.988 & 0.953 & 0.952 \\
\hline & & 6 & 0 & 0.944 & 0.959 & 0.971 & 0.963 & 0.994 & 0.981 & 0.995 & 0.990 & 0.989 & 0.992 & 0.960 & 0.957 \\
\hline \multirow[t]{12}{*}{300} & 0.3 & 3 & 0 & 0.943 & 0.953 & 0.958 & 0.957 & 0.960 & 0.942 & 0.950 & 0.926 & 0.969 & 0.957 & 0.927 & 0.927 \\
\hline & & 4 & 0 & 0.944 & 0.954 & 0.960 & 0.959 & 0.977 & 0.958 & 0.976 & 0.955 & 0.983 & 0.975 & 0.940 & 0.940 \\
\hline & & 5 & 0 & 0.943 & 0.955 & 0.961 & 0.958 & 0.983 & 0.963 & 0.991 & 0.971 & 0.994 & 0.987 & 0.950 & 0.951 \\
\hline & & 6 & 0 & 0.943 & 0.957 & 0.961 & 0.957 & 0.985 & 0.964 & 0.996 & 0.978 & 0.997 & 0.991 & 0.954 & 0.954 \\
\hline & 0.5 & 3 & 0 & 0.954 & 0.956 & 0.965 & 0.966 & 0.961 & 0.963 & 0.951 & 0.952 & 0.975 & 0.975 & 0.933 & 0.931 \\
\hline & & 4 & 0 & 0.956 & 0.958 & 0.965 & 0.967 & 0.977 & 0.979 & 0.976 & 0.978 & 0.986 & 0.987 & 0.946 & 0.949 \\
\hline & & 5 & 0 & 0.958 & 0.960 & 0.965 & 0.967 & 0.980 & 0.983 & 0.991 & 0.992 & 0.995 & 0.995 & 0.955 & 0.960 \\
\hline & & 6 & 0 & 0.959 & 0.961 & 0.964 & 0.967 & 0.981 & 0.983 & 0.996 & 0.996 & 0.998 & 0.999 & 0.958 & 0.964 \\
\hline & 0.7 & 3 & 0 & 0.940 & 0.954 & 0.959 & 0.957 & 0.962 & 0.944 & 0.953 & 0.931 & 0.971 & 0.962 & 0.925 & 0.925 \\
\hline & & 4 & 0 & 0.941 & 0.954 & 0.960 & 0.959 & 0.977 & 0.960 & 0.974 & 0.955 & 0.984 & 0.975 & 0.938 & 0.939 \\
\hline & & 5 & 0 & 0.942 & 0.954 & 0.961 & 0.958 & 0.982 & 0.964 & 0.989 & 0.972 & 0.993 & 0.986 & 0.948 & 0.947 \\
\hline & & 6 & 0 & 0.943 & 0.955 & 0.961 & 0.958 & 0.983 & 0.965 & 0.993 & 0.977 & 0.997 & 0.990 & 0.954 & 0.952 \\
\hline \multicolumn{16}{|c|}{ Panel B. Length } \\
\hline \multirow[t]{12}{*}{150} & 0.3 & 3 & 0 & 0.066 & 0.078 & 0.296 & 0.277 & 0.654 & 0.611 & 0.729 & 0.714 & 0.736 & 0.726 & 0.682 & 0.648 \\
\hline & & 4 & 0 & 0.046 & 0.050 & 0.147 & 0.145 & 0.586 & 0.528 & 0.709 & 0.659 & 0.707 & 0.648 & 0.644 & 0.557 \\
\hline & & 5 & 0 & 0.035 & 0.035 & 0.078 & 0.082 & 0.493 & 0.417 & 0.649 & 0.526 & 0.637 & 0.492 & 0.565 & 0.400 \\
\hline & & 6 & 0 & 0.029 & 0.026 & 0.051 & 0.055 & 0.387 & 0.294 & 0.545 & 0.348 & 0.525 & 0.301 & 0.452 & 0.230 \\
\hline & 0.5 & 3 & 0 & 0.064 & 0.060 & 0.228 & 0.172 & 0.632 & 0.571 & 0.732 & 0.707 & 0.744 & 0.724 & 0.691 & 0.644 \\
\hline & & 4 & 0 & 0.048 & 0.041 & 0.108 & 0.084 & 0.557 & 0.469 & 0.714 & 0.643 & 0.723 & 0.643 & 0.662 & 0.550 \\
\hline & & 5 & 0 & 0.038 & 0.029 & 0.066 & 0.055 & 0.459 & 0.341 & 0.663 & 0.505 & 0.667 & 0.487 & 0.595 & 0.396 \\
\hline & & 6 & 0 & 0.031 & 0.022 & 0.049 & 0.040 & 0.353 & 0.216 & 0.566 & 0.328 & 0.564 & 0.302 & 0.491 & 0.231 \\
\hline & 0.7 & 3 & 0 & 0.069 & 0.081 & 0.297 & 0.278 & 0.648 & 0.604 & 0.725 & 0.710 & 0.734 & 0.723 & 0.684 & 0.649 \\
\hline & & 4 & 0 & 0.048 & 0.051 & 0.148 & 0.147 & 0.581 & 0.523 & 0.703 & 0.652 & 0.702 & 0.642 & 0.644 & 0.554 \\
\hline & & 5 & 0 & 0.035 & 0.034 & 0.077 & 0.081 & 0.490 & 0.415 & 0.642 & 0.520 & 0.633 & 0.485 & 0.564 & 0.395 \\
\hline & & 6 & 0 & 0.028 & 0.025 & 0.049 & 0.054 & 0.384 & 0.293 & 0.541 & 0.344 & 0.520 & 0.295 & 0.449 & 0.227 \\
\hline 300 & 0.3 & 3 & 0 & 0.039 & 0.047 & 0.134 & 0.143 & 0.588 & 0.538 & 0.713 & 0.677 & 0.765 & 0.757 & 0.722 & 0.715 \\
\hline & & 4 & 0 & 0.030 & 0.033 & 0.064 & 0.075 & 0.460 & 0.413 & 0.687 & 0.646 & 0.765 & 0.756 & 0.712 & 0.690 \\
\hline & & 5 & 0 & 0.026 & 0.025 & 0.045 & 0.051 & 0.328 & 0.298 & 0.648 & 0.599 & 0.754 & 0.734 & 0.688 & 0.633 \\
\hline & & 6 & 0 & 0.021 & 0.019 & 0.034 & 0.038 & 0.221 & 0.207 & 0.593 & 0.532 & 0.729 & 0.666 & 0.641 & 0.524 \\
\hline & 0.5 & 3 & 0 & 0.045 & 0.044 & 0.097 & 0.087 & 0.549 & 0.467 & 0.704 & 0.658 & 0.765 & 0.755 & 0.724 & 0.712 \\
\hline & & 4 & 0 & 0.035 & 0.030 & 0.062 & 0.056 & 0.397 & 0.309 & 0.670 & 0.610 & 0.764 & 0.749 & 0.717 & 0.686 \\
\hline & & 5 & 0 & 0.029 & 0.021 & 0.045 & 0.038 & 0.257 & 0.188 & 0.621 & 0.547 & 0.756 & 0.721 & 0.698 & 0.626 \\
\hline & & 6 & 0 & 0.025 & 0.015 & 0.035 & 0.029 & 0.163 & 0.117 & 0.563 & 0.465 & 0.739 & 0.646 & 0.662 & 0.519 \\
\hline & 0.7 & 3 & 0 & 0.051 & 0.060 & 0.137 & 0.147 & 0.592 & 0.541 & 0.712 & 0.675 & 0.764 & 0.756 & 0.722 & 0.714 \\
\hline & & 4 & 0 & 0.036 & 0.038 & 0.066 & 0.077 & 0.463 & 0.416 & 0.686 & 0.643 & 0.762 & 0.753 & 0.712 & 0.691 \\
\hline & & 5 & 0 & 0.028 & 0.026 & 0.045 & 0.052 & 0.330 & 0.300 & 0.645 & 0.596 & 0.752 & 0.733 & 0.687 & 0.631 \\
\hline & & 6 & 0 & 0.023 & 0.019 & 0.034 & 0.038 & 0.223 & 0.209 & 0.589 & 0.528 & 0.727 & 0.667 & 0.641 & 0.525 \\
\hline
\end{tabular}


Table 4. Finite sample coverage and length of nominal 0.95-level confidence sets.

\begin{tabular}{|c|c|c|c|c|c|c|c|c|c|c|c|c|c|c|c|}
\hline \multirow[b]{2}{*}{$T$} & \multirow[b]{2}{*}{$\tau_{0}$} & \multirow[b]{2}{*}{$\delta_{1}$} & \multirow[b]{2}{*}{$\delta_{2}$} & \multicolumn{2}{|c|}{$\rho=0.00$} & \multicolumn{2}{|c|}{$\rho=0.50$} & \multicolumn{2}{|c|}{$\rho=0.80$} & \multicolumn{2}{|c|}{$\rho=0.90$} & \multicolumn{2}{|c|}{$\rho=0.95$} & \multicolumn{2}{|c|}{$\rho=1.00$} \\
\hline & & & & $S$ & $S^{*}$ & $S$ & $S^{*}$ & $S$ & $S^{*}$ & $S$ & $S^{*}$ & $S$ & $S^{*}$ & $S$ & $S^{*}$ \\
\hline \multicolumn{16}{|c|}{ Panel A. Coverage } \\
\hline \multirow[t]{12}{*}{150} & 0.3 & 0 & 0.3 & 0.928 & 0.954 & 0.952 & 0.956 & 0.968 & 0.962 & 0.965 & 0.973 & 0.943 & 0.962 & 0.881 & 0.881 \\
\hline & & 0 & 0.4 & 0.930 & 0.956 & 0.955 & 0.960 & 0.978 & 0.974 & 0.977 & 0.983 & 0.951 & 0.970 & 0.891 & 0.893 \\
\hline & & 0 & 0.5 & 0.931 & 0.954 & 0.958 & 0.960 & 0.985 & 0.978 & 0.983 & 0.988 & 0.959 & 0.977 & 0.902 & 0.905 \\
\hline & & 0 & 0.6 & 0.935 & 0.957 & 0.961 & 0.962 & 0.987 & 0.982 & 0.986 & 0.992 & 0.964 & 0.982 & 0.912 & 0.915 \\
\hline & 0.5 & 0 & 0.3 & 0.948 & 0.945 & 0.958 & 0.955 & 0.971 & 0.971 & 0.975 & 0.970 & 0.958 & 0.940 & 0.886 & 0.847 \\
\hline & & 0 & 0.4 & 0.948 & 0.947 & 0.962 & 0.959 & 0.980 & 0.981 & 0.986 & 0.981 & 0.968 & 0.953 & 0.898 & 0.864 \\
\hline & & 0 & 0.5 & 0.951 & 0.949 & 0.965 & 0.964 & 0.985 & 0.986 & 0.990 & 0.987 & 0.975 & 0.963 & 0.907 & 0.881 \\
\hline & & 0 & 0.6 & 0.951 & 0.950 & 0.966 & 0.965 & 0.988 & 0.989 & 0.993 & 0.990 & 0.979 & 0.970 & 0.917 & 0.895 \\
\hline & 0.7 & 0 & 0.3 & 0.925 & 0.950 & 0.950 & 0.957 & 0.965 & 0.960 & 0.963 & 0.968 & 0.946 & 0.968 & 0.883 & 0.883 \\
\hline & & 0 & 0.4 & 0.925 & 0.952 & 0.954 & 0.958 & 0.976 & 0.970 & 0.974 & 0.979 & 0.956 & 0.975 & 0.894 & 0.896 \\
\hline & & 0 & 0.5 & 0.928 & 0.953 & 0.956 & 0.960 & 0.982 & 0.974 & 0.981 & 0.987 & 0.962 & 0.981 & 0.904 & 0.907 \\
\hline & & 0 & 0.6 & 0.929 & 0.955 & 0.959 & 0.962 & 0.985 & 0.977 & 0.985 & 0.990 & 0.968 & 0.986 & 0.913 & 0.918 \\
\hline \multirow[t]{12}{*}{300} & 0.3 & 0 & 0.3 & 0.934 & 0.952 & 0.957 & 0.958 & 0.981 & 0.965 & 0.989 & 0.977 & 0.989 & 0.989 & 0.920 & 0.922 \\
\hline & & 0 & 0.4 & 0.936 & 0.953 & 0.956 & 0.959 & 0.984 & 0.966 & 0.994 & 0.981 & 0.993 & 0.992 & 0.928 & 0.929 \\
\hline & & 0 & 0.5 & 0.935 & 0.953 & 0.957 & 0.960 & 0.985 & 0.966 & 0.995 & 0.983 & 0.995 & 0.994 & 0.934 & 0.935 \\
\hline & & 0 & 0.6 & 0.936 & 0.954 & 0.958 & 0.960 & 0.986 & 0.967 & 0.996 & 0.984 & 0.996 & 0.995 & 0.936 & 0.938 \\
\hline & 0.5 & 0 & 0.3 & 0.955 & 0.953 & 0.963 & 0.965 & 0.975 & 0.976 & 0.987 & 0.988 & 0.991 & 0.991 & 0.920 & 0.907 \\
\hline & & 0 & 0.4 & 0.956 & 0.954 & 0.965 & 0.967 & 0.978 & 0.978 & 0.991 & 0.992 & 0.995 & 0.995 & 0.930 & 0.920 \\
\hline & & 0 & 0.5 & 0.957 & 0.955 & 0.966 & 0.967 & 0.980 & 0.981 & 0.995 & 0.995 & 0.997 & 0.997 & 0.937 & 0.928 \\
\hline & & 0 & 0.6 & 0.959 & 0.957 & 0.967 & 0.968 & 0.982 & 0.982 & 0.996 & 0.996 & 0.998 & 0.998 & 0.942 & 0.935 \\
\hline & 0.7 & 0 & 0.3 & 0.931 & 0.953 & 0.956 & 0.957 & 0.980 & 0.964 & 0.989 & 0.977 & 0.987 & 0.987 & 0.921 & 0.920 \\
\hline & & 0 & 0.4 & 0.931 & 0.954 & 0.959 & 0.960 & 0.982 & 0.966 & 0.992 & 0.981 & 0.992 & 0.992 & 0.927 & 0.928 \\
\hline & & 0 & 0.5 & 0.932 & 0.954 & 0.960 & 0.960 & 0.983 & 0.967 & 0.994 & 0.983 & 0.993 & 0.993 & 0.933 & 0.936 \\
\hline & & 0 & 0.6 & 0.931 & 0.954 & 0.959 & 0.961 & 0.983 & 0.968 & 0.995 & 0.983 & 0.995 & 0.994 & 0.938 & 0.939 \\
\hline \multicolumn{16}{|c|}{ Panel B. Length } \\
\hline \multirow[t]{12}{*}{150} & 0.3 & 0 & 0.3 & 0.130 & 0.134 & 0.187 & 0.188 & 0.385 & 0.370 & 0.624 & 0.602 & 0.659 & 0.627 & 0.605 & 0.565 \\
\hline & & 0 & 0.4 & 0.105 & 0.109 & 0.152 & 0.154 & 0.329 & 0.301 & 0.559 & 0.493 & 0.586 & 0.520 & 0.539 & 0.486 \\
\hline & & 0 & 0.5 & 0.089 & 0.094 & 0.128 & 0.133 & 0.271 & 0.244 & 0.464 & 0.378 & 0.489 & 0.409 & 0.464 & 0.404 \\
\hline & & 0 & 0.6 & 0.079 & 0.084 & 0.113 & 0.119 & 0.222 & 0.208 & 0.360 & 0.299 & 0.388 & 0.321 & 0.386 & 0.330 \\
\hline & 0.5 & 0 & 0.3 & 0.136 & 0.118 & 0.186 & 0.167 & 0.362 & 0.335 & 0.608 & 0.572 & 0.653 & 0.602 & 0.604 & 0.548 \\
\hline & & 0 & 0.4 & 0.111 & 0.096 & 0.152 & 0.137 & 0.311 & 0.266 & 0.546 & 0.453 & 0.581 & 0.483 & 0.536 & 0.459 \\
\hline & & 0 & 0.5 & 0.092 & 0.083 & 0.128 & 0.118 & 0.263 & 0.214 & 0.455 & 0.333 & 0.484 & 0.365 & 0.458 & 0.370 \\
\hline & & 0 & 0.6 & 0.078 & 0.074 & 0.111 & 0.105 & 0.217 & 0.181 & 0.355 & 0.255 & 0.383 & 0.278 & 0.379 & 0.294 \\
\hline & 0.7 & 0 & 0.3 & 0.133 & 0.135 & 0.189 & 0.189 & 0.380 & 0.363 & 0.614 & 0.587 & 0.651 & 0.618 & 0.606 & 0.564 \\
\hline & & 0 & 0.4 & 0.108 & 0.110 & 0.153 & 0.154 & 0.324 & 0.297 & 0.548 & 0.487 & 0.578 & 0.518 & 0.539 & 0.484 \\
\hline & & 0 & 0.5 & 0.091 & 0.095 & 0.129 & 0.133 & 0.270 & 0.244 & 0.458 & 0.381 & 0.487 & 0.414 & 0.463 & 0.402 \\
\hline & & 0 & 0.6 & 0.080 & 0.084 & 0.113 & 0.118 & 0.222 & 0.206 & 0.364 & 0.303 & 0.394 & 0.329 & 0.386 & 0.327 \\
\hline 300 & 0.3 & 0 & 0.3 & 0.067 & 0.072 & 0.097 & 0.103 & 0.174 & 0.174 & 0.344 & 0.313 & 0.589 & 0.518 & 0.545 & 0.502 \\
\hline & & 0 & 0.4 & 0.056 & 0.061 & 0.080 & 0.087 & 0.140 & 0.143 & 0.258 & 0.234 & 0.425 & 0.352 & 0.429 & 0.384 \\
\hline & & 0 & 0.5 & 0.049 & 0.053 & 0.069 & 0.076 & 0.118 & 0.123 & 0.198 & 0.193 & 0.292 & 0.268 & 0.322 & 0.288 \\
\hline & & 0 & 0.6 & 0.044 & 0.048 & 0.062 & 0.068 & 0.105 & 0.110 & 0.166 & 0.166 & 0.225 & 0.217 & 0.241 & 0.223 \\
\hline & 0.5 & 0 & 0.3 & 0.072 & 0.065 & 0.098 & 0.091 & 0.168 & 0.155 & 0.331 & 0.284 & 0.570 & 0.474 & 0.539 & 0.475 \\
\hline & & 0 & 0.4 & 0.056 & 0.054 & 0.080 & 0.077 & 0.136 & 0.126 & 0.252 & 0.208 & 0.416 & 0.309 & 0.421 & 0.348 \\
\hline & & 0 & 0.5 & 0.048 & 0.047 & 0.070 & 0.067 & 0.116 & 0.110 & 0.195 & 0.169 & 0.290 & 0.226 & 0.314 & 0.251 \\
\hline & & 0 & 0.6 & 0.043 & 0.043 & 0.062 & 0.061 & 0.103 & 0.098 & 0.163 & 0.144 & 0.222 & 0.181 & 0.235 & 0.189 \\
\hline & 0.7 & 0 & 0.3 & 0.072 & 0.075 & 0.098 & 0.103 & 0.174 & 0.173 & 0.342 & 0.314 & 0.580 & 0.517 & 0.550 & 0.506 \\
\hline & & 0 & 0.4 & 0.059 & 0.062 & 0.080 & 0.086 & 0.139 & 0.142 & 0.260 & 0.236 & 0.430 & 0.362 & 0.433 & 0.386 \\
\hline & & 0 & 0.5 & 0.051 & 0.054 & 0.070 & 0.076 & 0.118 & 0.123 & 0.199 & 0.193 & 0.300 & 0.271 & 0.325 & 0.289 \\
\hline & & 0 & 0.6 & 0.045 & 0.049 & 0.062 & 0.068 & 0.104 & 0.110 & 0.166 & 0.166 & 0.228 & 0.218 & 0.243 & 0.223 \\
\hline
\end{tabular}

